\title{
Magnetic resonance cholangiopancreatography: the ABC of MRCP
}

\author{
Nyree Griffin • Geoff Charles-Edwards • \\ Lee Alexander Grant
}

Received: 30 May 2011 / Accepted: 9 September 2011 /Published online: 28 September 2011

(C) European Society of Radiology 2011

\begin{abstract}
Magnetic resonance cholangiopancreatography (MRCP) is a technique that has evolved over the past two decades. It continues to have a fundamental role in the noninvasive investigation of many pancreatico-biliary disorders. The purpose of this review is to summarise the key concepts behind MRCP, the different techniques that are currently employed (including functional and secretin-stimulated MRCP), the pitfalls the reader should be aware of, and the main clinical indications for its use.
\end{abstract}

Keywords MR cholangiopancreatography - Bile duct calculi - Bile duct neoplasms · Bile duct abnormalities . Pancreatic ducts

\section{Introduction}

It has been exactly two decades since magnetic resonance cholangiopancreatography (MRCP) was first described [1]. Over this time, the technique has evolved considerably, aided by improvements in spatial resolution and speed of

\section{N. Griffin $(\square)$}

Department of Radiology, St Thomas' Hospital,

Westminster Bridge Road,

London SE1 7EH, UK

e-mail: nyree.griffin@gstt.nhs.uk

G. Charles-Edwards

Department of Medical Physics, St Thomas' Hospital,

Westminster Bridge Road,

London SE1 7EH, UK

L. A. Grant

Department of Radiology, Royal Free Hospital,

Pond Street,

London NW3 2QG, UK acquisition. It has now an established role in the investigation of many biliary disorders, serving as a non-invasive alternative to endoscopic retrograde cholangiopancreatography (ERCP). It makes use of heavily $\mathrm{T} 2$-weighted pulse sequences, thus exploiting the inherent differences in the T2-weighted contrast between stationary fluid-filled structures in the abdomen (which have a long T2 relaxation time) and adjacent soft tissue (which has a much shorter T2 relaxation time). Static or slow moving fluids within the biliary tree and pancreatic duct appear of high signal intensity on MRCP, whilst surrounding tissue is of reduced signal intensity.

Heavily T2-weighted images were originally achieved using a gradient-echo (GRE) balanced steady-state free precession technique $[1,2]$. A fast spin-echo (FSE) pulse sequence with a long echo time (TE) was then introduced shortly after [3], with the advantages of a higher signal-tonoise ratio and contrast-to-noise ratio, and a lower sensitivity to motion and susceptibility artefacts. Modified FSE sequences have been described, including rapid acquisition with rapid enhancement (RARE) [4], half-Fourier acquisition single-shot turbo spin-echo (HASTE) [5], and fast-recovery fast spin-echo (FRFSE) [6] sequences. Both breath-hold (using a single shot approach) [7] and non-breath-hold techniques (with respiratory triggering) [8] have been used, with images obtained either as a two-dimensional (2D) or three-dimensional (3D) acquisition. A 3D technique provides a higher signal to noise ratio, which is traded off for thinner contiguous slices. Acquiring images with near isotropic voxels allows improved post-processing manipulation of the images with multi-planar reconstruction, maximum intensity projection (MIP) and volume rendering. The introduction of faster gradients and a parallel acquisition technique has resulted in even greater spatial resolution and faster acquisition times. More recently, functional assessment of biliary excretion and pancreatic exocrine function has 
Table 1 Summary of MRCP imaging parameters

\begin{tabular}{|c|c|c|c|}
\hline Parameter & $\begin{array}{l}\text { T2-weighted breath-hold HASTE } \\
\text { (liver down to ampulla) }\end{array}$ & $\begin{array}{l}\text { 3D T2-weighted FSE } \\
\text { with respiratory triggering }\end{array}$ & $\begin{array}{l}\text { T2 weighted breath-hold HASTE } \\
\text { fat-saturated thick slab }\end{array}$ \\
\hline TR/TE (ms) & $1,000 / 83$ & $1,800 / 678$ & $4,500 / 752$ \\
\hline Number of averages & 1 & 1 & 1 \\
\hline Flip angle & 150 & 180 & 180 \\
\hline Field of view (mm) & $350 \times 263$ & $380 \times 380$ & $350 \times 350$ \\
\hline Matrix size & $256 \times 146$ & $384 \times 380$ & $384 \times 300$ \\
\hline Slice thickness (mm) & $7 \mathrm{~mm}$ & $1.5 \mathrm{~mm}$ & $40 \mathrm{~mm}$ \\
\hline Slice gap (mm) & $0.7 \mathrm{~mm}$ & $0 \mathrm{~mm}$ & N/A \\
\hline Number of slices & 20 & 40 & 1 \\
\hline Acquisition plane & Axial & Coronal oblique & Coronal \\
\hline Half-Fourier factor & $5 / 8$ & $\begin{array}{l}\text { Phase-encoding: off } \\
\text { Slice-encoding: } 6 / 8\end{array}$ & Phase encoding: $7 / 8$ \\
\hline Parallel imaging acceleration factor & 2 & 2 & 2 \\
\hline Receiver bandwidth (Hz/pixel) & 391 & 260 & 150 \\
\hline Turbo factor & 146 & 127 & 307 \\
\hline Oversampling & None & Slice: $20 \%$ & Phase: $33 \%$ \\
\hline
\end{tabular}

HASTE half-Fourier acquisition single shot turbo spin echo, FSE fast spin echo

become possible with the use of hepatobiliary contrast media [9] and secretin [10] respectively.

The purpose of this pictorial review is to describe (1) the MRCP protocol used by our centre and additional/alterna- tive sequences which can be employed, (2) the normal biliary anatomy on MRCP, (3) the potential pitfalls associated with this technique and (4) the main clinical indications for its use.
Fig. 1 Example of a normal secretin-stimulated MRCP in a patient being investigated for sphincter of Oddi dysfunction, with idiopathic dilatation of the CBD. a Thick coronal slab at baseline shows the pancreatic duct is of normal caliber (arrow) with little secretions in the duodenum $(D)$; b) at $3 \mathrm{~min}$ there is progressive filling of the duodenum $(D)$ due to an increase in pancreatic secretions and the pancreatic duct has become more prominent (arrow); c) at $9 \mathrm{~min}$, the pancreatic duct has returned to baseline calibre (arrow)
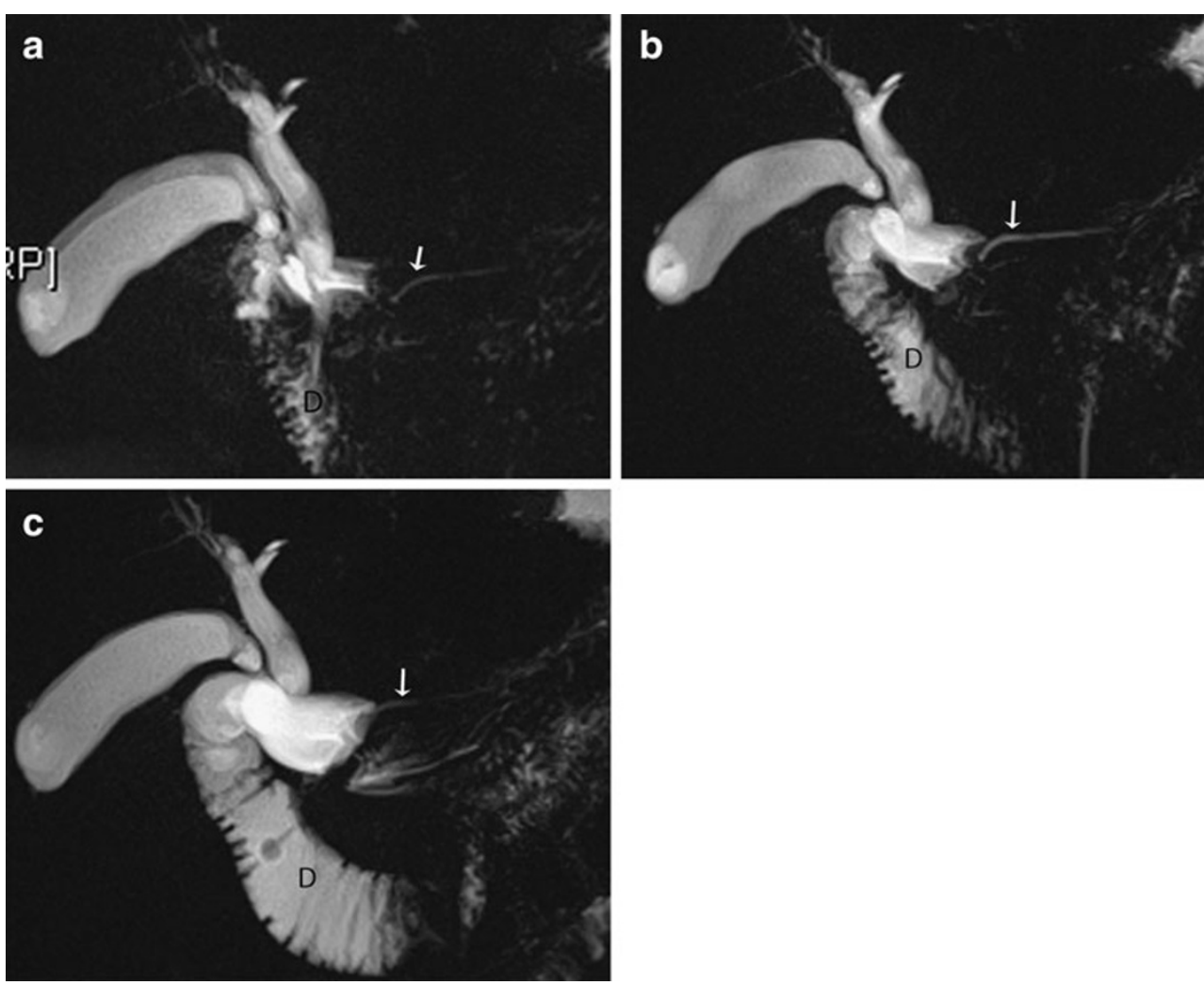


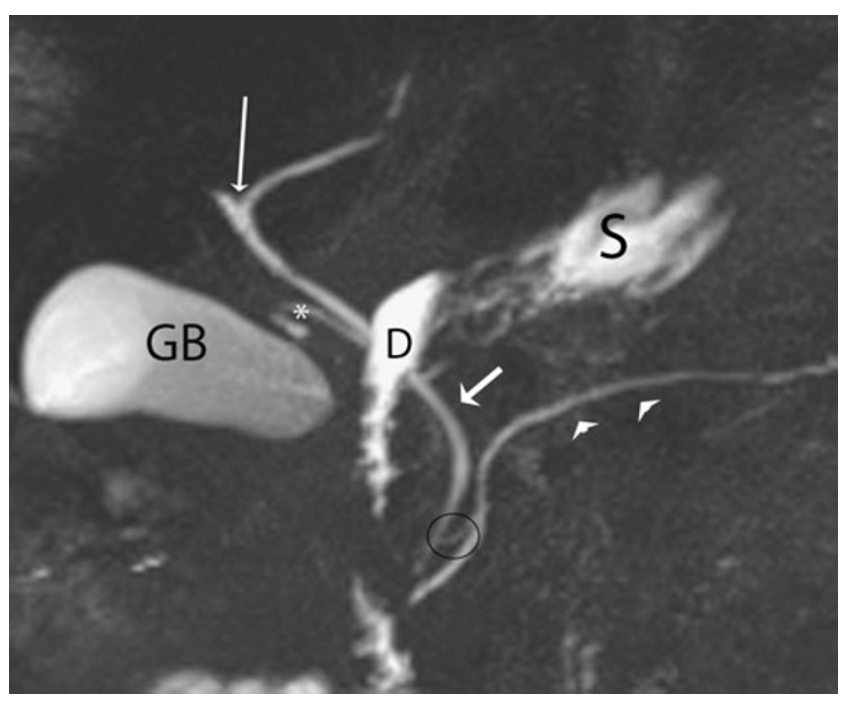

Fig. 2 Normal anatomy on MRCP. The confluence of the right and left intrahepatic ducts to form the common hepatic duct is seen (long thin arrow). The cystic duct (*) typically joins the right side of the common hepatic duct to form the common bile duct (CBD) (short arrow). The main pancreatic duct (arrowheads) drains along with the CBD into the major duodenal papilla. An accessory pancreatic duct is present (black circle), draining into the minor duodenal papilla. Fluid containing structures such as the gallbladder $(G B)$, duodenum $(D)$ and stomach $(S)$ are well seen on this T2-weighted sequence with the duodenum obscuring part of the biliary tree

\section{MRCP protocol}

Patients are fasted for $4 \mathrm{~h}$ prior to the study in order to reduce fluid secretions within the stomach and duodenum, reduce bowel peristalsis and promote gallbladder distension. We do not routinely use an anti-peristaltic agent. Some centres use a negative oral contrast agent (e.g. iron oxide or blueberry juice) to reduce the signal intensity of overlapping fluid within the stomach and duodenum, although this is not part of our routine protocol.
At our institution, MRCP is performed on a 1.5-T Avanto MRI system (Siemens, Erlangen, Germany), using a phasedarray body coil. The protocol imaging parameters are shown in Table 1. We first perform an axial 2D breath-hold HASTE sequence. Two breath-hold acquisitions are obtained, so that the whole of the liver down to the duodenal ampulla is visualised.

Following this, we perform two 3D respiratory-triggered heavily T2-weighted FSE sequences in the coronal oblique plane. The imaging plane is selected from the initial axial T2-weighted images, with one acquisition aligned to the common bile duct (CBD) in the head of the pancreas and the second acquisition aligned to the pancreatic duct at approximately 90 degrees to the first imaging plane. Respiratory triggering is achieved with the use of a navigator sequence that employs an MR pre-pulse to monitor respiratory motion. The navigator is placed over the edge of the diaphragm on the coronal and sagittal localisers and image acquisition is triggered when the position of this diaphragm interface with the lung falls within a pre-specified acceptance window. In this way a consistent position of the imaging slice is obtained. The patient is asked to breathe regularly throughout this acquisition, which takes between 3-5 min to acquire. A stack of 40 slices are obtained, which are contiguous and each of $1.5 \mathrm{~mm}$ in thickness. As the images are heavily $\mathrm{T} 2$-weighted, the pancreatico-biliary tree is displayed as high signal intensity, whilst adjacent structures are of reduced signal intensity. This sequence is useful in detecting small filling defects or strictures in the biliary or pancreatic ducts. From this volume of data, a MIP reformat can be generated. This displays only the pixel with the highest signal intensity along a ray perpendicular to the plane of projection. It thus highlights bile-filled and fluid-filled structures very well. MIP reformats can be generated in various coronal and sagittal oblique planes. We convention-
Fig. 3 Example of a partial voluming artefact. a Coronal maximum intensity projection (MIP) reformat shows a possible filling defect (arrow) in the dilated distal CBD. b The thinsection MRCP source image in fact demonstrates multiple filling defects (arrows) in the CBD, in keeping with stones
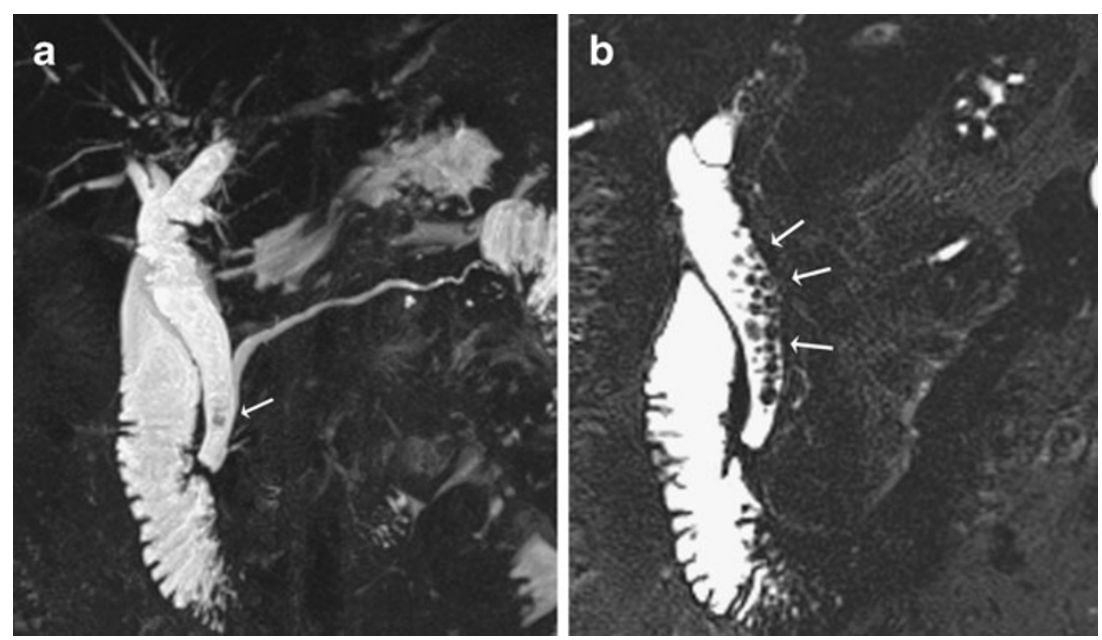

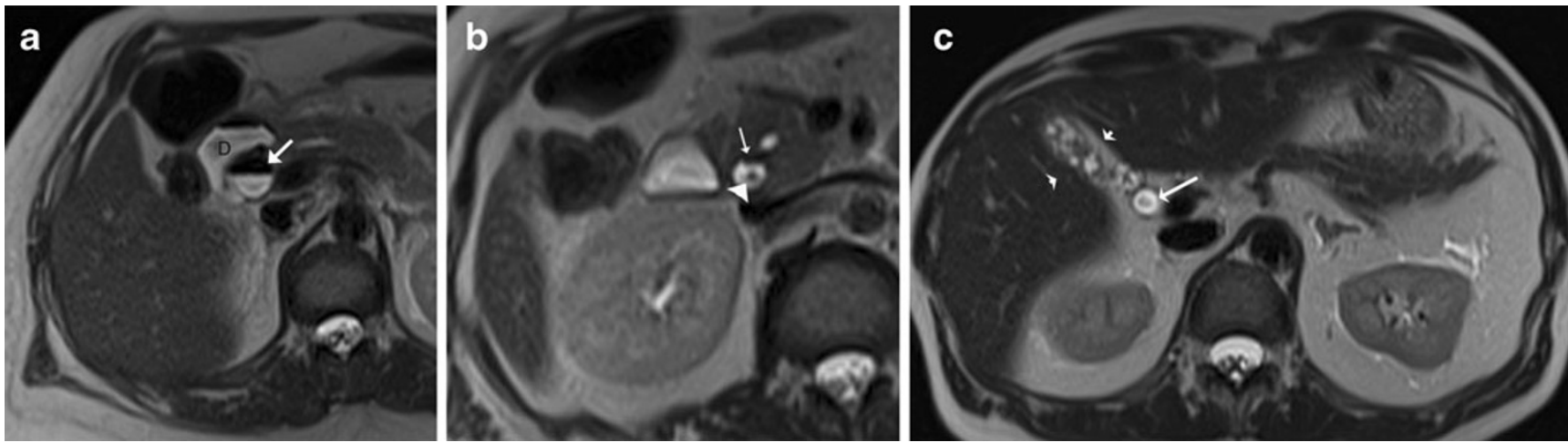

Fig. 4 Example of intra-ductal factors causing potential pitfalls in interpretation. a Axial T2-weighted MRI shows an air-fluid level in a dilated proximal CBD in keeping with aerobilia (arrow), adjacent to the duodenum $(D)$, which also shows an air-fluid level. b More distally in the same patient, the cause of the obstruction is seen with a dependent filling defect (arrowhead) in the distal CBD in keeping

ally create 18 MIP reformats at 10-degree intervals to each other over a radial array of 180 degrees.

In addition to, or as an alternative to the MIP reformats, a thick collimation slab can be obtained in the coronal plane. This involves performing a fat saturated HASTE sequence where a single slab of data $4 \mathrm{~cm}$ in thickness is acquired in a 1to 2-s breath-hold. It is useful in depicting the entire pancreatico-biliary tree and no post-processing is required.

In order to evaluate the duct walls, and any focal parenchymal pathology, 3D fat suppressed T1-weighted GRE sequences before and after intravenous contrast administration can also be performed.

\section{Secretin-stimulated MRCP}

Secretin is an endogenous hormone normally produced by the duodenum, which stimulates exocrine secretion of the with a stone. This should not be confused with the non-dependent aerobilia also shown at this level (arrow). c Axial T2-weighted MRI in a different patient shows a central filling defect in a dilated CBD which is due to flow artefact (arrow). The patient also has chronic cholecystitis with a contracted gallbladder (arrowheads)

pancreas. When given as a synthetic agent intravenously ( $1 \mathrm{ml} / 10 \mathrm{~kg}$ body weight), it improves the visualisation of the pancreatic duct by increasing its calibre. Pancreatic juice flows out of the major duodenal papilla to progressively fill the duodenum. We perform a thick slab MRCP in the coronal oblique plane at baseline and then at 1, 3, 5, 7 and $9 \mathrm{~min}$ following injection. Its effect starts almost immediately and peaks between 2 to 5 mins. By $10 \mathrm{~min}$, the calibre of the main pancreatic duct should return to baseline (Fig. 1a-c) with persistent dilatation of $>3 \mathrm{~mm}$ considered abnormal. The indications for this technique include the detection and characterisation of pancreatic ductal anomalies and strictures, evaluation of the integrity of the pancreatic duct, characterisation of any communication between the pancreatic duct and pseudocysts/pancreatic fistulas, and the assessment of pancreatic function and sphincter of Oddi dysfunction.

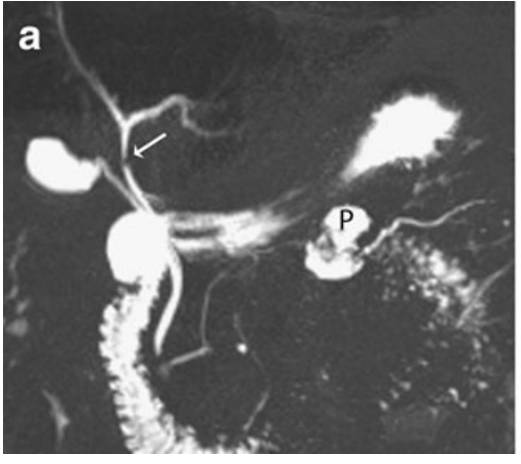

Fig. 5 a Example of an extra-ductal factor causing a potential pitfall in interpretation. Coronal MIP reformat suggests a stricture or possible filling defect in the common hepatic duct (arrow) but with no upstream dilatation. Incidental note is also made of a small pseudocyst $(P)$ associated with the main pancreatic duct due to a history of

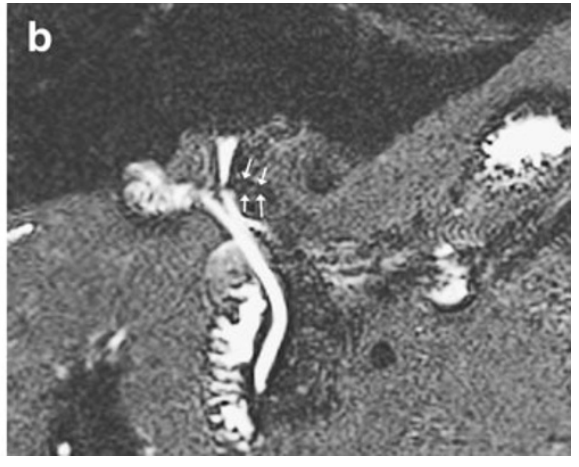

pancreatitis. b Thin-section MRCP image more clearly shows that this is due to extrinsic compression from the right hepatic artery which appears as a subtle curvilinear signal void outside the duct and extending across it (arrows) 
Functional MR cholangiography

This involves the use of MR lipophilic paramagnetic contrast agents, which when given intravenously, show hepato-biliary excretion. Contrast agents include gadobenate dimeglumine (Gd-BOPTA, Multihance; Bracco Imaging, Milan, Italy), gadolinium ethoxybenzyldiethylenetriamine penta-acetic acid (Gd-EOB-DTPA, Primovist; Bayer-Schering Pharma, Berlin, Germany) and, historically, mangafodopir trisodium (Teslascan; GE Healthcare, Oslo, Norway). Delayed imaging in the axial and coronal plane, performed between 10-120 min following intravenous administration, normally results in hyper-intense bile on 3D T1-weighted fat-saturated GRE images. The signalto-noise ratio is higher than conventional T2-weighted MRCP, allowing better delineation of the bile ducts. This technique can be used for similar indications as for T2-weighted MRCP and in most cases has a similar diagnostic accuracy. It is more expensive than conventional T2-weighted MRCP and only the biliary tree is depicted. For these reasons, most centres continue to use conventional T2-weighted MRCP. However, functional MRCP does have a number of advantages, as follows: (1) it better demonstrates communications between cystic lesions and draining bile ducts in the diagnosis of congenital biliary disorders (e.g. Caroli's disease) [11], (2) it helps to distinguish true obstruction in a dilated biliary system (where delayed or no biliary excretion is demonstrated) from pseudo-obstruction [9], and (3) it can demonstrate active extravasation of contrast in suspected bile leaks $[12,13]$. Another advantage is that these gadolinium-based hepatobiliary-specific contrast agents initially distribute in the extracellular fluid compartment, thus allowing for early dynamic pre-contrast and post-contrast images in the arterial, portal venous and equilibrium phase prior to the functional cholangiogram.

In cases where there is significant biliary obstruction or impaired hepatocyte function, delayed images up to 24 $\mathrm{h}$ can be performed until contrast is seen in the gallbladder and duodenum.

\section{Normal anatomy on MRCP}

Only central intra-hepatic bile ducts are normally seen on MRCP (Fig. 2), usually measuring up to $3 \mathrm{~mm}$ in diameter, whilst extra-hepatic bile ducts should not exceed $7 \mathrm{~mm}$. In patients with a previous cholecystectomy, mild biliary dilatation occurs, with the CBD measuring up to $10 \mathrm{~mm}$ in diameter. The intra-hepatic biliary drainage system parallels the portal venous supply. The right hepatic duct has two major branches: the right posterior duct, which has an almost horizontal course (draining posterior segments VI and VII), and the right anterior duct, which has a more vertical course (draining the anterior segments V and VIII). The right posterior duct usually runs posterior to the right

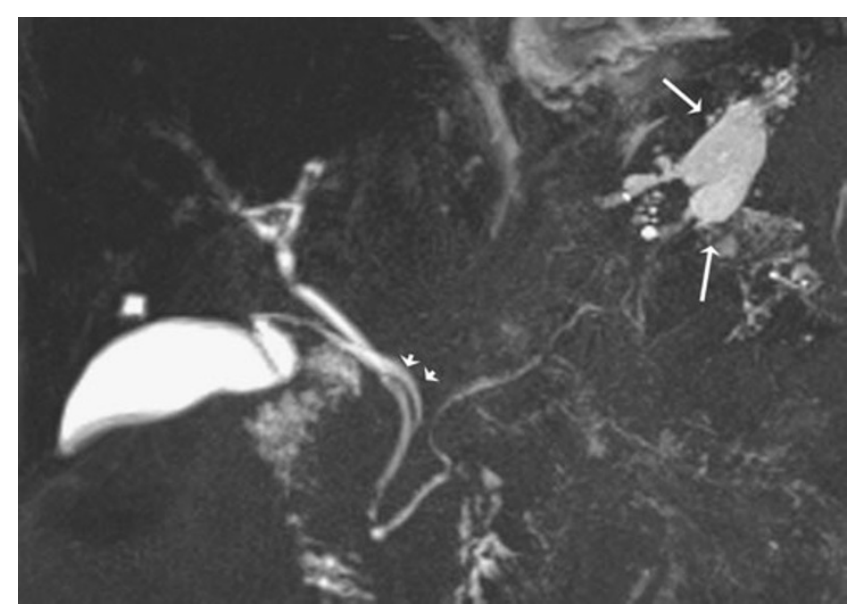

Fig. 6 Example of a normal cystic duct variant. Coronal MIP reformat shows medial insertion of the cystic duct into the common duct (short arrows). There is also a pseudocyst in the lesser sac due to chronic pancreatitis (long arrows)

anterior duct and fuses with it on its left (medial) side. The left hepatic duct drains segments II-IV and joins the right hepatic duct to form the common hepatic duct. Segment 1 drains via a separate bile duct usually into the origin of the left or right hepatic duct.

The pancreatic duct should be no greater than $3 \mathrm{~mm}$, with the main pancreatic duct of Wirsung normally draining into the major duodenal papilla along with the CBD (91\% of individuals). An accessory pancreatic duct of Santorini may be present in $45 \%$ [14], which drains into the minor duodenal papilla. The cystic duct usually joins the extrahepatic duct from the right lateral aspect in $50 \%$ of cases, although it may insert into its anterior or posterior aspect in $30 \%$ and medial aspect in $20 \%$ of individuals.

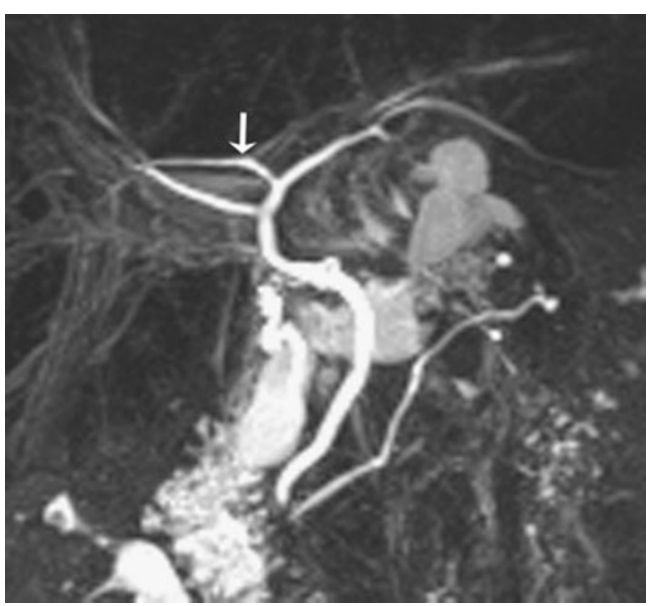

Fig. 7 Example of variant intrahepatic bile duct branching. Coronal MIP reformat shows the commonest type, where the right posterior sectoral duct (arrow) drains into the left hepatic duct. Note its more horizontal orientation compared to the right anterior hepatic duct 


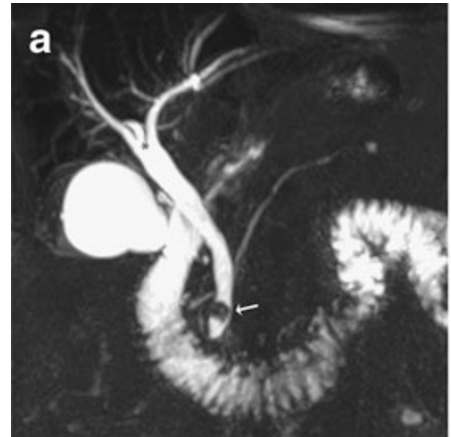

Fig. 8 a Coronal MIP reformat shows a triple confluence $(*)$ of right anterior and posterior hepatic ducts and left hepatic duct to form the common hepatic duct. A CBD stone is seen as a low signal filling defect in the distal CBD (arrow) with mild upstream dilatation. b

\section{Pitfalls on MRCP}

A number of pitfalls may arise which fall into four main categories: (1) artefacts related to technique and reconstruction; (2) normal variants mimicking pathology; (3) intra-ductal factors; (4) extra-ductal factors.

\section{Technique and reconstruction artefacts}

A thick slab MRCP may obscure small filling defects or strictures as the spatial resolution is degraded because of volume averaging effects. Partial volume effects also degrade spatial resolution in MIP reformats, leading to the missed filling defects (Fig. 3a, b) and over- or underestimation of strictures. Due to respiratory motion artefact, the biliary tree may appear stenotic, dilated, disconnected or duplicated on MIP reformats. Hence it is important to always review the original thin-section data set also.

\section{Normal variants}

A long cystic duct running parallel to the CBD may simulate a dilated common duct, whilst a contracted choledochal sphincter may mimic an impacted stone or stricture in the distal CBD. En face visualisation of the cystic duct insertion into the bile duct may also simulate a filling defect. Performing MRCP in multiple imaging planes or carrying out repeat MRCP imaging will help resolve these problems.

\section{Intra-ductal factors}

Filling defects in the bile may arise, not only from bile duct calculi but also from the presence of gas, debris, haemorrhage and tumour. Aerobilia is seen as a non-dependent filling defect on the axial images (Fig. 4a, b), whilst a signal void in the central part of the bile duct is due to flow
Axial T2-weighted MRI of a different patient shows oedematous gallbladder wall in keeping with acute cholecystitis (short arrow) with multiple small dependent filling defects in the CBD in keeping with bile duct stones (long thin arrow)

phenomenon (Fig. 4c) and may occur in dilated ducts and at the point of insertion of a large cystic duct. The presence of iodinated contrast material will also reduce the signal intensity of bile.

\section{Extra-ductal factors}

Pulsatile vascular compression from adjacent vessels may mimic a stricture. The commonest site of extrinsic vascular compression is the common hepatic duct, followed by the left hepatic duct, both due to the right hepatic artery crossing its posterior aspect (Fig. 5a, b). The mid portion of the CBD may also be narrowed due to the gastro-duodenal artery. Pseudo-obstruction is typically seen as a bandlike compression with minimal proximal dilatation. Susceptibility artefacts from metallic clips and gas may give rise to difficulties in interpretation, although titanium clips used

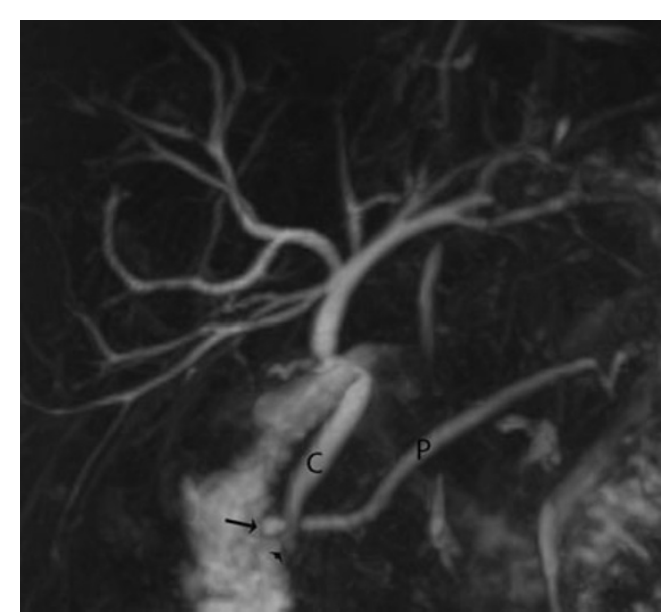

Fig. 9 Example of a pancreas divisum. Coronal MIP reformat shows the main pancreatic duct $(P)$ draining into the minor duodenal papilla (arrow). The CBD $(C)$ drains more inferiorly into the major duodenal papilla (arrowhead). The accessory duct is not well seen on this MIP 
Fig. 10 a Coronal MIP reformat shows early primary sclerosing cholangitis (PSC) with irregular dilatation and strictures seen in the left sided intrahepatic ducts (arrows). b Coronal MIP reformat in a different patient with more advanced PSC with multiple intrahepatic strictures and strictures seen in the common hepatic duct and distal CBD (arrows). There is dilatation of the proximal CBD
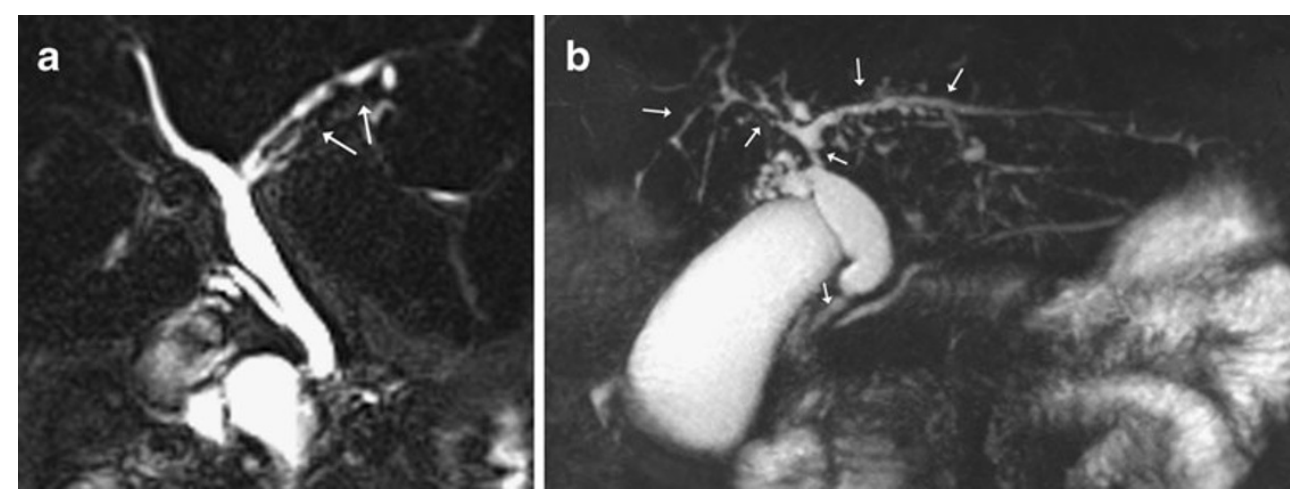

nowadays for cholecystectomy are not magnetic. Overlapping of the biliary tree with other stationary fluids (i.e. from adjacent bowel, cystic collections or ascites) may also cause interpretation problems.

\section{Clinical indications for MRCP}

Identification of congenital anomalies of the cystic and hepatic ducts

A normal biliary anatomy is present in only about $60 \%$ of the population. There are many cystic duct variants $[15,16]$, including a low insertion, a medial insertion (Fig. 6), and drainage of the cystic duct into the right hepatic duct. The most important variant of intrahepatic bile duct branching clinically is the presence of an aberrant right posterior sectoral duct draining into the common hepatic duct or cystic duct in $5 \%$ of the population. This should be recognised on MRCP (if performed), as it may be ligated or cut at the time of cholecystectomy. The most common anatomic variant of intrahepatic bile duct branching, occurring in $13-19 \%$ of the population $[15,17]$ is drainage of the right anterior or posterior duct into the left hepatic duct (Fig. 7). This is

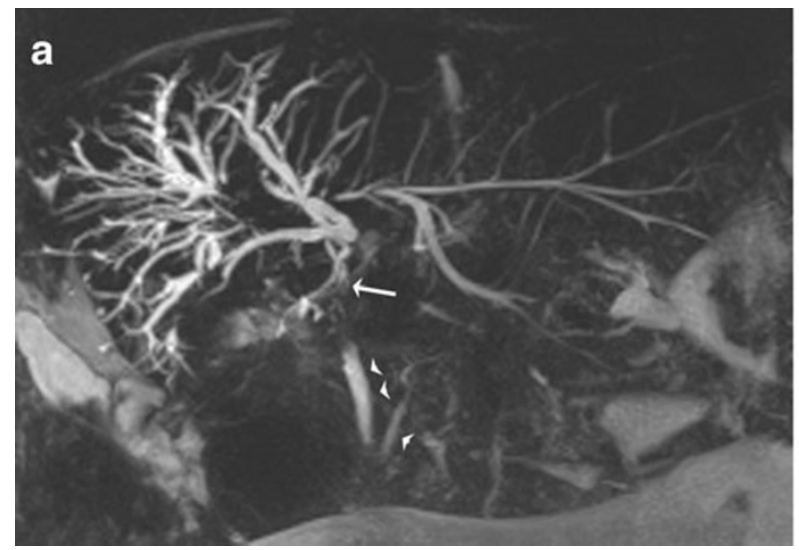

Fig. 11 a Coronal MIP reformat shows dilatation of the intrahepatic bile ducts with disconnection between the left and right sided ducts and the common duct, due to a hilar cholangiocarcinoma (arrow). The

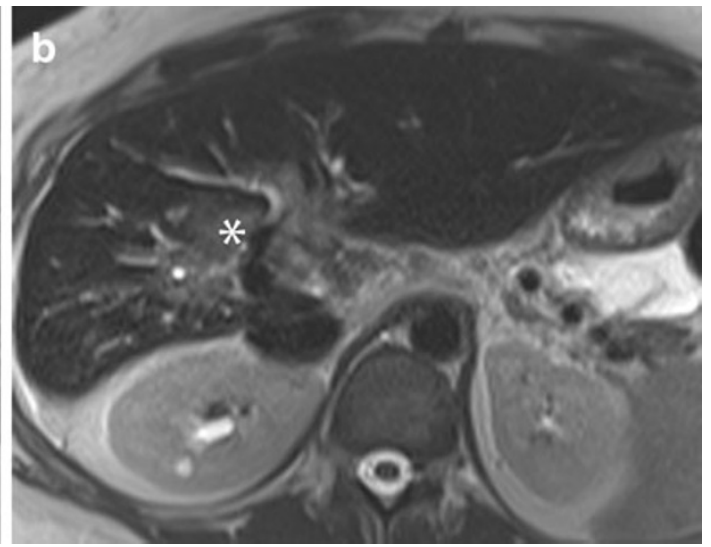

important to detect in patients undergoing left hepatectomy for living related transplant donation as accidental ligation/ resection may lead to cirrhosis of segments 5 and 8 or segments 6 and 7 respectively. A triple confluence [15] may also be seen $(11 \%)$ where the right posterior duct, right anterior duct and left hepatic duct simultaneously drain into the common hepatic duct (Fig. 8a). When ERCP is used as the 'gold standard', MRCP has an accuracy of between 90 to $95 \%$ in the diagnosis of such congenital anomalies [16].

Post-surgical biliary anatomy and complications

ERCP is often not possible in patients with a previous biliary-enteric anastomosis. MRCP is then useful in the demonstration of post-surgical biliary anatomy and in the detection of biliary complications, with a $100 \%$ sensitivity in the diagnosis of anastomotic strictures and a $90 \%$ sensitivity for choledocholithiasis [18].

\section{Pancreas divisum}

This is the most common pancreatic variant and is associated with an increased prevalence of acute pancreatitis. There is

distal CBD and pancreatic duct appear normal (arrowheads). b Corresponding axial T2-weighted image shows high signal intensity tumour at the porta hepatis $(*)$ 
failure of fusion of the dorsal and ventral pancreatic ducts. The larger dominant dorsal pancreatic duct drains the tail, body and superior head of the pancreas into the minor duodenal papilla (Fig. 9), whilst the smaller ventral duct drains the inferior pancreatic head and uncinate process into the major duodenal papilla, along with the CBD. MRCP has a $100 \%$ accuracy in the detection of this condition [19].

Anomalous pancreaticobiliary junction

Choledochal cysts are associated with anomalous union of the pancreaticobiliary duct, where the pancreatic duct and CBD unite outside the duodenal wall and form a long common channel greater than $15 \mathrm{~mm}$ in length. There are five different types of choledochal cyst described (Todani classification) with three main types of anomalous pancreaticobiliary junction. MRCP can assist detection of such variants when suspected clinically.

\section{Choledocholithiasis}

This is usually performed in patients presenting with obstructive liver function tests and with suspected gallstones and/or a dilated CBD on ultrasound. It is also carried out in patients who have persistent symptoms and abnormal liver function following cholecystectomy. A systematic review of the literature has shown that when compared with ERCP, MRCP has an aggregated sensitivity, specificity, positive predictive value and negative predictive value of $85 \%, 93 \%, 87 \%$, and $82 \%$ respectively [20]. Stones (as small as $2 \mathrm{~mm}$ ) appear as dependent low-signal-filling defects within the CBD (Fig. 8a, b), surrounded by highsignal-intensity bile.

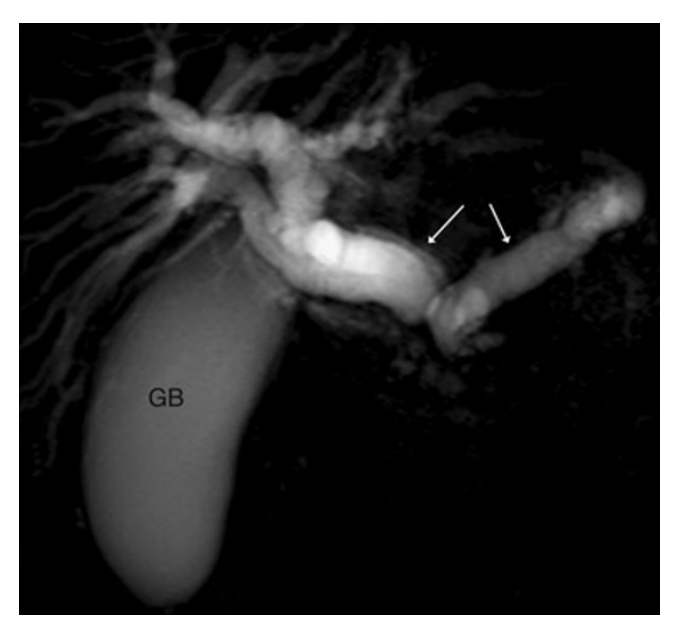

Fig. 12 Coronal thick slab MRCP shows the classical 'double duct' sign in a patient with carcinoma at the head of pancreas with dilatation of both the CBD and pancreatic duct (arrows) and distension of the gallbladder $(G B)$

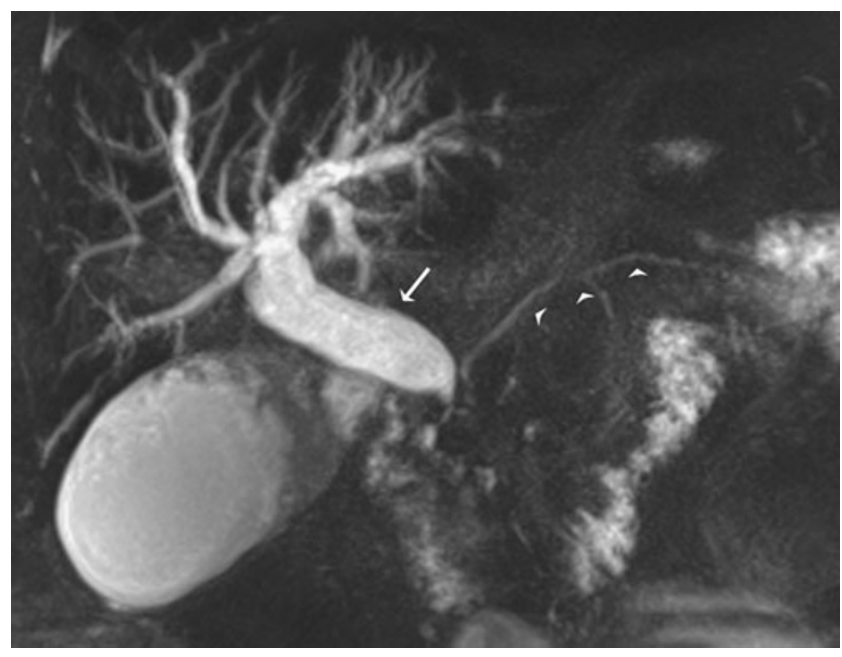

Fig. 13 Coronal MIP reformat shows intrahepatic bile duct dilatation and a grossly dilated CBD (arrow) with abrupt distal termination due to a periampullary tumour. There is no pancreatic duct dilatation (arrowheads)

Benign biliary strictures

These usually develop following surgical injury (95\%) from procedures such as laparoscopic cholecystectomy, hepatic resection, liver transplantation and biliary enteric anastomosis. Other causes include trauma, inflammation from choledocholithiasis, ischaemia involving the hepatic artery and primary sclerosing cholangitis (PSC). Typically a benign stricture involves a short segment, with a regular margin and symmetric narrowing. MRCP can demonstrate the site and extent of the stricture with a reported sensitivity of $91-100 \%$ [21].

PSC is a fibrosing inflammatory process of the bile ducts, resulting in stenosis of both intrahepatic $(80 \%)$ and extrahepatic ducts. Seventy percent of patients have inflammatory bowel disease [22] and $10 \%$ of patients with

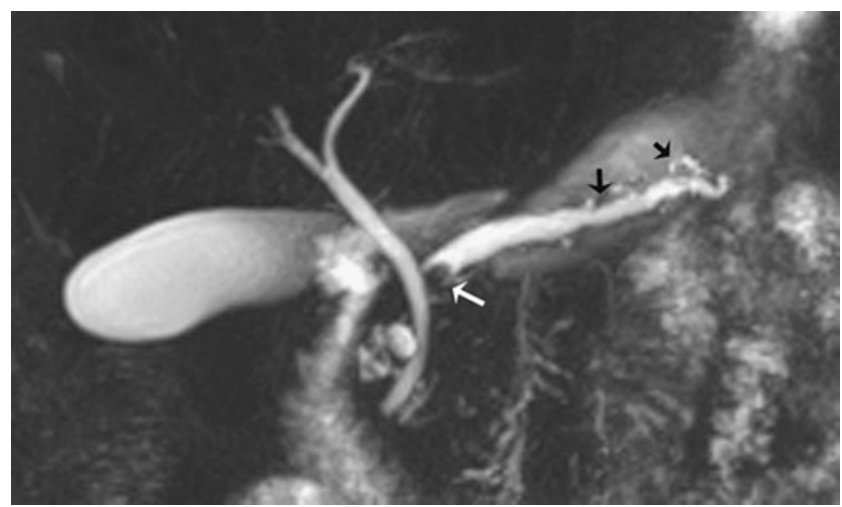

Fig. 14 Coronal MIP reformat in a patient with chronic pancreatitis shows dilatation of the main pancreatic duct, ectasia of the side branches (black arrows) and a stone in the proximal pancreatic duct (white arrow) 

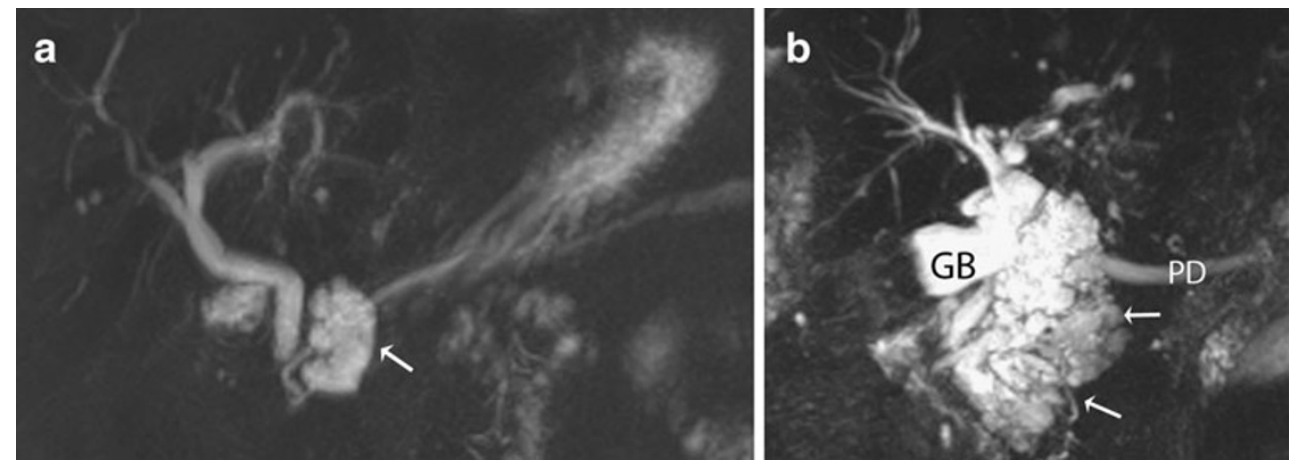

Fig. 15 a Coronal MIP reformat shows a small multi-septated cystic lesion (arrow) arising in the uncinate process of the pancreas with communication with the main pancreatic duct, in keeping with a side branch intraductal papillary mucinous neoplasm (IPMN). b Coronal

PSC will develop a cholangiocarcinoma. MRCP is not as sensitive as ERCP in the detection of early changes, but is useful for follow-up in established cases, in order to determine severity and to recognise the development of complications. On MRCP, the ducts appear beaded or have a 'pruned tree' appearance with multifocal strictures demonstrated, with intervening normal or slightly dilated ducts seen (Fig. 10a, b).

\section{Malignant biliary strictures}

Cholangiocarcinoma is a tumour arising from the bile ducts. On MRCP the periductal type is seen as a biliary stricture,
MIP reformat in a different patient, shows a large ill defined multiseptated cystic lesion (arrows) arising in the region of the head/uncinate process of the pancreas separate to the gallbladder $(G B)$ with pancreatic duct $(P D)$ dilatation in keeping with a mixed main and side branch IPMN

involving the $\mathrm{CBD}$, common hepatic duct, biliary bifurcation (Klatskin tumour) or intrahepatic ducts. Proximal bile duct dilatation occurs (Fig. 11a). Suspicious features include increased wall thickness $(>3 \mathrm{~mm})$, increased signal intensity on T2-weighted images (Fig. 11b) and progressive enhancement of the bile ducts (due to the fibrotic component of the tumour) following intravenous administration of gadolinium. A malignant extrahepatic bile duct stricture is likely to be longer than a benign stricture, with an irregular margin and asymmetric narrowing.

Pancreatic adenocarcinoma usually appears as a focal mass, most often in the head of the pancreas, leading to

Table 2 Differences in epidemiology and morphology on MRCP of cystic pancreatic tumours

\begin{tabular}{|c|c|c|c|}
\hline & Serous cystadenoma & Mucinous cystic neoplasms & Intraductal mucinous neoplasm (IPMN) \\
\hline Demographics & $\begin{array}{l}\text { Typically older } \\
\text { women }>60 \text { years }\end{array}$ & $\begin{array}{l}\text { Typically younger } \\
\text { women } 30-50 \text { years }\end{array}$ & Peak age 6th decade, no gender bias \\
\hline Site of tumour & $\begin{array}{l}\text { Anywhere in the pancreas, } \\
\text { especially the head }\end{array}$ & $75 \%$ in body/tail & $\begin{array}{l}\text { Side branch type: usually pancreatic } \\
\text { head/uncinate process, less frequently } \\
\text { in the tail; tumour communicates with } \\
\text { the main pancreatic duct } \\
\text { Main duct type: segmental or diffuse } \\
\text { involvement of the main pancreatic duct }\end{array}$ \\
\hline Morphology & $\begin{array}{l}>6 \text { cysts }(<2 \mathrm{~cm} \text { each }), \\
\text { thin septations, central } \\
\text { scar (calcification), does } \\
\text { not communicate with } \\
\text { the pancreatic duct }\end{array}$ & $\begin{array}{l}\text { Cysts }>2 \mathrm{~cm} \text {, unilocular } \\
\text { or multilocular, does } \\
\text { not communicate with } \\
\text { the pancreatic duct }\end{array}$ & $\begin{array}{l}\text { Side branch type: macrocystic or microcystic } \\
\text { appearances }\end{array}$ \\
\hline & & $\begin{array}{l}\text { Features of malignancy on MR } \\
\text { denoted by thick septations, } \\
\text { soft tissue nodules, and/or } \\
\text { pancreatic duct dilatation }\end{array}$ & $\begin{array}{l}\text { Main duct type: diffuse duct dilatation due } \\
\text { to gross mucin production, micropapillary } \\
\text { studding, pancreatic atrophy }\end{array}$ \\
\hline Average size & $5 \mathrm{~cm}$ & $6-10 \mathrm{~cm}$ & Larger size with malignant tumours \\
\hline $\begin{array}{l}\text { Signal } \\
\text { characteristics }\end{array}$ & Fluid signal & $\begin{array}{l}\text { High signal intensity on } \mathrm{T} 1 \\
\text { and } \mathrm{T} 2 \text { (mucin/blood) }\end{array}$ & $\begin{array}{l}\text { High signal intensity on } \\
\text { T1 (mucin), intermediate } \\
\text { signal intensity on T2 }\end{array}$ \\
\hline Comments & Usually benign & Malignant in $50 \%$ & $\begin{array}{l}\text { Side branch type: usually } \\
\text { associated with benign adenomas } \\
\text { Main duct type: malignant in } 40 \%\end{array}$ \\
\hline
\end{tabular}


encasement and obstruction of the pancreatic duct and/or CBD. Dilatation of both ducts is seen in approximately $75 \%$ of cases appearing as the 'double duct' sign on MRCP (Fig. 12). Although contrast-enhanced CT is conventionally used for staging local and distant spread in pancreatic cancers, MRCP may be used as an initial investigation in identifying the level of obstruction in patients presenting with painless obstructive jaundice.

Peri-ampullary carcinoma can lead to high-grade obstruction of the CBD with abrupt termination on MRCP (Fig. 13). There is usually only mild dilatation of the pancreatic duct.

\section{Chronic pancreatitis}

Chronic inflammation of the pancreas results in parenchymal destruction with fibrosis, fat necrosis and dystrophic calcification. Strictures in the main pancreatic duct may eventually develop a 'chain-of-lakes' appearance with alternating stenoses and dilatation. Side branch ectasia and intraductal calculi occur (Fig. 14). In advanced cases there can be marked dilatation of both the pancreatic duct and CBD simulating the 'double duct' sign seen with carcinoma of the head of pancreas. The sensitivity of MRCP in the detection of early side branch changes can be increased with the use of secretin-stimulated MRCP [23].

Pseudocysts are encapsulated fluid collections seen in both acute and chronic pancreatitis (Figs. 5, 6). These often develop within the lesser sac. MRCP is more sensitive than ERCP in demonstrating these fluid collections and may show their connection with the pancreatic duct. With ERCP, less than $50 \%$ of pseudocysts opacify with contrast [24].

\section{Cystic pancreatic tumours}

Cystic pancreatic tumours include serous cystadenomas, mucinous cystic neoplasms and intraductal papillary mucinous neoplasm (IPMN). Often, small cystic lesions within the pancreas are detected incidentally on CT performed for other indications. MRCP can help delineate these tumours more clearly (Fig. 15a, b). Table 2 shows the difference in epidemiology and MRCP morphology between these cystic tumours.

\section{Biliary injuries}

Following transection of the bile ducts (usually following surgery), bile accumulates within the sub-hepatic space. Fluid collections can be appreciated on MRCP with transection of the affected bile duct. If functional MRCP is used, extravasation of contrast from the biliary tree is seen. A stricture may develop following accidental ligation or transection, with upstream dilatation demonstrated on MRCP.

\section{Conclusion}

The technique of MRCP has evolved considerably over the last 2 decades, with technological advances in both acquisition and post processing. It remains the investigation of choice for the non-invasive diagnosis of many pancreatico-biliary disorders. It is hoped that this review has helped remind the reader as to the basic concepts behind MRCP, the different sequences that can now be employed, the pitfalls one should be aware of, and why, even in modern day, it remains a test fit for purpose in the radiological investigation of biliary pathology.

\section{References}

1. Wallner BK, Schumacher KA, Weidenmaier W, Friedrich JM (1991) Dilated biliary tract: evaluation with MR cholangiography with a T2-weighted contrast-enhanced fast sequence. Radiology 181:805-808

2. Morimoto K, Shimoi M, Shirakawa T et al (1992) Biliary obstruction: evaluation with three-dimensional MR cholangiography. Radiology 183:578-580

3. Outwater EK (1993) MR cholangiography with a fast spin-echo sequence. J Magn Reson Imaging 3(P):131

4. Laubenberger J, Buchert M, Schneider B, Blum U, Hennig J, Langer M (1995) Breath-hold projection magnetic resonance cholangiopancreaticography (MRCP): a new method for the examination of the bile and pancreatic ducts. Magn Reson Med 33:18-23

5. Miyazaki T, Yamashita Y, Tsuchigame T, Yamamoto H, Urata J, Takahashi M (1996) MR cholangiopancreatography using HASTE (half-Fourier acquisition single-shot turbo spin-echo) sequences. AJR Am J Roentgenol 166:1297-1303

6. Sodickson A, Mortele KJ, Barish MA, Zou KH, Thibodeau S, Tempany CMC (2006) Three-dimensional fast-recovery fast spin-echo MRCP: comparison with two-dimensional single shot fast spin echo techniques. Radiology 238:549-559

7. Takehara Y, Ichijo K, Tooyama N et al (1994) Breath-hold MR cholangiopancreatography with a long-echo-train fast spin-echo sequence and a surface coil in chronic pancreatitis. Radiology 192:73-78

8. Barish MA, Yucel EK, Soto JA, Chuttani R, Ferrucci JT (1995) MR Cholangiopancreatography: efficacy of three-dimensional turbo spin-echo technique. AJR Am J Roentgenol 165:295-300

9. Fayad LM, Holland GA, Bergin D et al (2003) Functional magnetic resonance cholangiography (fMRC) of the gallbladder and biliary tree with contrast-enhanced magnetic resonance cholangiography. J Magn Reson Imaging 18:449-460

10. Baillie J, Kimberly J (2007) Prospective comparison of secretinstimulated MRCP with manometry in the diagnosis of sphincter of Oddi dysfunction types II and III. Gut 56:742-744

11. Park MS, Yu JS, Lee JH, Kim KW (2007) Value of manganeseenhanced T1- and T2-weighted MR cholangiography for differentiating cystic parenchymal lesions from cystic abnormalities which communicate with bile ducts. Yonsei Med J 48:1072-1074

12. Aduna M, Larena JA, Martin D, Martinez-Guerenu B, Aguirre I, Astigarraga E (2005) Bile duct leaks after laparoscopic cholecystectomy: value of contrast-enhanced MRCP. Abdom Imaging 30:480-487

13. Thurley PD, Dhingsa R (2008) Laparoscopic cholecystectomy: postoperative imaging. AJR Am J Roentgenol 191:794-801 
14. Vitellas KM, Keogan MT, Spritzer CE, Nelson RC (2000) MR cholangiopancreatography of bile and pancreatic duct abnormalities with emphasis on the single-shot fast spin echo technique. Radiographics 20:939-957

15. Puente SG, Bannura GC (1983) Radiological anatomy of the biliary tract: variations and congenital abnormalities. World J Surg 7:271-276

16. Taourel P, Bret PM, Reinhold C, Barkun AN, Atri M (1996) Anatomic variants of the biliary tree: diagnosis with MR cholangiopancreatography. Radiology 199:521-527

17. Gazelle GS, Lee MJ, Mueller PR (1994) Cholangiographic segmental anatomy of the liver. Radiographics 14:1005-1013

18. Pavone P, Laghi A, Catalano C et al (1997) MR cholangiography in the examination of patients with biliary-enteric anastomoses. AJR Am J Roentgenol 169:807-811

19. Bret PM, Reinhold C, Taourel P, Guibaud L, Atri M, Barkun AN (1996) Pancreas divisum: evaluation with MR cholangiopancreatography. Radiology 199:99-103
20. Verma D, Kapadia A, Eisen GM, Adler DG (2006) EUS vs MRCP for detection of choledocholithiasis. Gastrointest Endosc 64:248-254

21. Lee MG, Lee HJ, Kim MH, Kang EM, Kim YH, Lee SG, Kim PN, Ha HK, Auh YH (1997) Extrahepatic biliary diseases: 3D MR cholangiopancreatography compared with endoscopic retrograde cholangiopancreatography. Radiology 202:663-669

22. Wiesner RH, LaRusso NF (1980) Clinicopathologic features of the syndrome of primary sclerosing cholangitis. Gastroenterology 79:200-206

23. Czako L (2007) Diagnosis of early-stage chronic pancreatitis by secretin-enhanced magnetic resonance cholangiopancreatography. J Gastroenterol 42:113-117

24. Nealon WH, Townsend CM Jr, Thompson JC (1989) Preoperative endoscopic retrograde cholangiopancreatography (ERCP) in patients with pancreatic pseudocyst associated with resolving acute and chronic pancreatitis. Ann Surg 209:532-538, discussion 538-540 